\title{
Effect of the Young modulus variability on the mechanical behaviour of a nuclear containment vessel
}

\author{
T. de Larrard ${ }^{\mathrm{a}}$, J.B. Colliat ${ }^{\mathrm{a}}$, F. Benboudjema ${ }^{\mathrm{a}}$, J.M. Torrenti ${ }^{\mathrm{b}}$, G. Nahas ${ }^{\mathrm{c}}$ \\ ${ }^{a}$ LMT-ENS Cachan, CNRS/UPMC/PRES UniverSud Paris, France \\ ${ }^{b}$ Université Paris-Est, LCPC, France \\ ${ }^{c} I R S N / D S R / S A M S / B A G S$, Fontenay-aux-Roses, France
}

\begin{abstract}
This study aims at investigating the influence of the Young modulus variability on the mechanical behaviour of a nuclear containment vessel in case of a loss of cooling agent accident and under the assumption of an elastic behaviour. To achieve this investigation, the Monte-Carlo Method is carried out thanks to a middleware which encapsulates the different components (random field generation, FE simulations) and enables calculations parallelization. The main goal is to quantify the uncertainty propagation by comparing the maximal values of outputs of interest (orthoradial stress and Mazars equivalent strain) with the ones obtained from a deterministic calculation. The Young modulus is supposed to be accurately represented by a weakly homogeneous random field and realizations are provided through its - troncated - Karhunen-Loève expansion. This study reveals a significant increase of the maximal equivalent strain if the Young modulus variability is considered (compared to a deterministic approach). The influence of the correlation length is investigated too. Finally it is shown that there is no correlation between the maximal values location of equivalent strain and the ones where the Young modulus extreme values are observed.
\end{abstract}


Keywords: Uncertainty propagation, Young modulus, Mazars equivalent strain, Nuclear containment vessel, Loss of coolant accident, Monte-Carlo Method, Karhunen-Loève expansion, Random fields

\section{Introduction}

Civil engineering constructions are naturally subjected to variability, from many origins: their dimensions, their construction processes, their exposure to several solicitations... Among these variables, which are influent on the behaviour of the structure and, thus, on its lifespan, some of them introduce a variability on the materials properties. Such uncertainties may evolve in time and be heterogeneously distributed inside the whole structure. As an example, Figure 1 shows the compressive strength distribution for the Millau viaduct's concrete (France). Although it is a high performance concrete and despite the care taken for the construction process, the observed variability is rather important, leading to a compressive strength range from $62 \mathrm{MPa}$ to almost $100 \mathrm{MPa}$. Because of its fabrication process, the variability of the its main constituents (cement, aggregates), its heterogeneous microstructure, the influence of its early-age history and the external environment (temperature, humidity, etc.), concrete is particularly subjected to variability. This is the main reason why the French National Research Agency set up the APPLET project, aiming at quantifying the variability of the characteristics of concrete, such as its mechanical properties or durability indicators (Poyet and Torrenti, 2010). The work presented in this paper is part of this project, and focuses on the Young modulus variability.

Dealing with nuclear containment vessels, it is worth noting that a large 


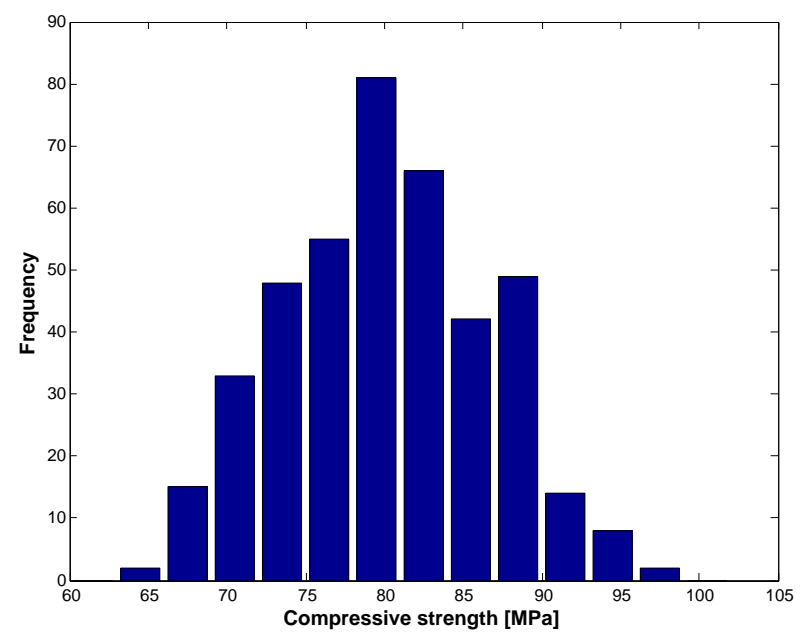

Figure 1: Compressive strength distribution of the Millau High Performance Concrete (source: Eiffage Company)

part of the French vessels have the feature to have no metallic liner, on the contrary of American structures for instance. Thus, concrete contributes to prevent from leaks and this is the reason why no major cracking can be tolerated. The design of the internal containment and of its pre-stress aims at preventing from any tensile strains when the structure is loaded so as to avoid cracking. Here we aim at investigating whether a spatial variation of the Young modulus in the containment vessel could lead the material to strain values for which cracking could occur.

Due to the always remaining uncertainties, it is usually not clear to what degree the results of numerical simulations match with reality. Focusing on any of those variabilities, the key issue is then to quantify the uncertainties propagation from the data to a set of chosen quantities of interest. For example an important question may be whether the scattering observed on 
any material parameter is enlarged when considering the ouputs. Within a numerical analysis framework, the data may be any of a Finite Element model parameter - like the elastic modulus here - and the quantities of interest chosen among any nodes displacements or Gauss points stresses. One fondamental issue may also be to compute the probability of a particular and most time rare - event to occur. Such fiability point of view is a major concern, especially within the nuclear engineering field.

Having set up a probabilistic model by reformulating the deterministic and possibly already discrete - problem within a chosen probability space, the question of stochastic integration leads to a large number of possible numerical approaches. Those approaches are usually divided into two classes (Spanos and Ghanem, 2002). On the one hand, the direct integration methods, which are mostly gathered into the so-called "Monte-Carlo" simulations (Metropolis and Ulam, 1949; Caflisch, 1998), are best viewed as numerical integration techniques. They require to solve many realizations of the deterministic problem and usually require a high computational effort. As an alternative, stochastic Galerkin methods aim at expanding the solution in a series of tensor products of spatial times random functions (Spanos and Ghanem, 2002; Keese, 2003). Once the coefficients of the series expansion are obtained, statistics may either be computed analytically or by sampling from the series expansion. They require to solve a possibly very large system of linear equations, which size grows exponentially along the number of stochastic dimensions and the stochastic interpolation degree. Although a lot of improvements have been achieved during the past two decades, such "curse of dimensions" still restrict the stochastic Galerkin approach to both linear 
and quite small size deterministic problems. Also considering the slow but independent from the number of dimesions convergence rate of the MonteCarlo methods, those are the main reasons why the presented work focuses on these stochastic numerical integration tools in order to quantify the Young modulus variability - viewed as a random field - propagation.

The outline of this paper is as follows: in the first part, we introduce the deterministic problem and discuss it. We also point out the quantities of interest. In the second part, we explain how the Karhunen-Loève expansion enables to generate random fields with the accurate properties. In the third part, we present the Monte-Carlo integration process and the numerical tool used for the probabilistic investigation. The last part is the discussion about the probabilistic simulations results and the influence of statistic parameters such as the correlation length.

\section{Mechanical deterministic problem}

The structure considered in this study is a concrete nuclear containment vessel for a pressurized water reactor (REP 1300 MW). The loading case corresponds to a loss of cooling agent accident: it results in an increase

of the pressure inside the vessel. In this first part we adopt a simplified deterministic model of such a structure and emphasize the chosen quantities of interest.

\subsection{Simplified structure modelling}

The containment vessel is here modeled as simply as possible in order to reduce the computational effort necessary to achieve a large number of simulations and ensure a good accuracy for the Monte-Carlo method. The 
structure itself has been considerably simplified to a cylinder of $54 \mathrm{~m}$ of height and $22.5 \mathrm{~m}$ of internal radius. The wall is $0.90 \mathrm{~m}$ thick. Finally the roof of the vessel is only modelled through an equivalent load (weight and internal pressure) on the top of the cylinder and, at the bottom of the cylinder, we consider the bottom slab (any other kind of boundary conditions induced unrealistic stress concentration in this area of the structure). The mechanical loading is assumed to be a $0.4 \mathrm{MPa}$ internal pressure. Moreover, we assume that the mechanical behaviour of the material remains elastic during the test. The initial state of stress is supposed to be equal to 0 (which is not the case due to restrained shrinkage at early-age - see for instance Benboudjema and Torrenti (2008)). Moreover, particular areas (like material and personal hatch) are not taken into account.

In order to compute the mechanical behaviour of the structure, the Finite Element code FEAP (Taylor and Zienkiewicz, 2005) is employed. The mesh is made of 108 elements in the height of the cylinder, and of 4 elements in the thickness of the wall and contains 200 orthoradial nodes. Figure 2 shows this very simplified modelling for the nuclear containment vessel. The major principal stress is plotted for a deterministic - constant Young modulus in the structure $(27 \mathrm{GPa})$ - simulation (the results are almost independent from the Young modulus value). Under such loading case, the major principle stress is very close to orthoradial stress.

\subsection{Outputs of interest: deterministic values}

In order to investigate whether the Young modulus variability could lead to a significant increase in the maximal values of stresses and strains, we aim at comparing the latter with their deterministic values. These values, 

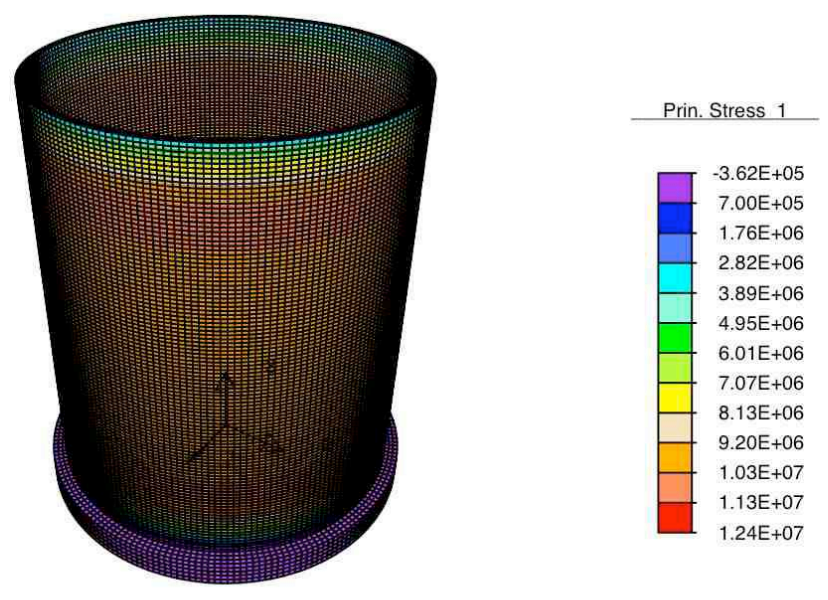

Figure 2: Major principal stress values $[\mathrm{Pa}]$ in the deterministic case

especially the orthoradial tensile stress, led to the prestress dimensioning. If the material reaches higher levels of tensile stress, cracking could occur, which is here assumed to be not acceptable. Therefore, it has been decided to focus on the maximal orthoradial stress and the maximal Mazars equivalent strain, calculated using the positive parts of the strains tensor (1). This equivalent strain is widely used for isotropic damage models and is a very common indicator to quantify concrete cracking risk (Mazars, 1986).

$$
\varepsilon_{e q}=\sqrt{\left\langle\varepsilon_{I}\right\rangle_{+}^{2}+\left\langle\varepsilon_{I I}\right\rangle_{+}^{2}+\left\langle\varepsilon_{I I I}\right\rangle_{+}^{2}}
$$

The reference values for these outputs of interest are given by a deterministic FE model with a modulus equal to $27 \mathrm{GPa}$. This value is also the expectation chosen for the modulus random field within the probabilistic setup (see §3). The maximal deterministic orthoradial stress in the vertical wall of the vessel (not considering the slab) is then equal to $10.6 \mathrm{MPa}$ and 
the maximal deterministic Mazars equivalent strain is $782 \cdot 10^{-6}$.

\section{Monte-Carlo integration and software engineering aspects}

In order to investigate the propagation of uncertainty from the input parameter (the Young modulus) to the output of interest (stresses and strains in the structure), a probabilistic approach is requested. Among the several existing methods, a direct integration method is chosen.

\subsection{Monte-Carlo Method}

Dealing with the computations of statistics for some quantities of interest, the Monte-Carlo fundamental principle is to estimate the integral - i.e the expectation - of this functional on some measure space through its evaluation for a finite number of realizations which are randomly chosen (Metropolis and Ulam, 1949; Caflisch, 1998). This idea appears in (2), where $\Psi$ denotes the functional that needs to be evaluated and which depends on the solution $u(\omega)$. In (2) $P(\omega)$ is the chosen probability measure, $N$ is the number of realization and the $\omega_{i}$ stand for the containment realizations. It is worth noting that despite its rather slow convergence, the Monte-Carlo method may be as accurate as required providing the number of "integration points" $N$ is large enough.

$$
\int_{\Omega} \Psi\left(\omega_{i}\right) d P(\omega)=\frac{1}{N} \sum_{i=1}^{N} \Psi\left(u\left(\omega_{i}\right)\right)+o\left(\frac{\sigma_{\Psi}^{2}}{N^{1 / 2}}\right)
$$

For every realization $\omega_{i}$, the $\Psi$ functional consists in solving a deterministic problem through the FE model presented in Part I. Those steps - which are the most time consuming - are all independent and thus can be made in 
parallel on a multi-processor computer or using a cluster. This point is the major advantage of the Monte-Carlo method and is certainly the main reason for its broad use. Finally, this direct integration process requires to generate $N$ independent realizations of the considered random field. Dealing with correlated random fields, it is necessary to employ the Karhunen-Loève expansion (Spanos and Ghanem, 2002), presented in $\S 4$. Before turning to this point, we stress on the software engineering importance within the context of Monte-Carlo methods.

\subsection{Software engineering aspects: component oriented software development}

The basic idea of component oriented software development is to separate tasks and to encapsulate them into components. Any encapsulated software is devoted to one task (for instance generating some Young modulus realizations or simulating the mechanical behaviour of the structure) and may be reused in different contexts (Szyperski, 1998). To some extend, such technology for software development can be viewed as an extension of object oriented programming for which each feature of a software can be encapsulated into a class. Classes, as well as components, can only be investigated through their interfaces and allow multiple instantiations of "objects".

Component oriented software development requires a so-called middleware which is a layer creating a communication framework between softwares. Among the different avalailable middlewares, the Components Template Library (CTL) developed by Matthies et al. (2006) is chosen. The main advantage of the latter is its very good performance dealing with scientific computing. Figure 3 shows a general picture describing the components architecture adopted in this work. The central part represents the Monte- 


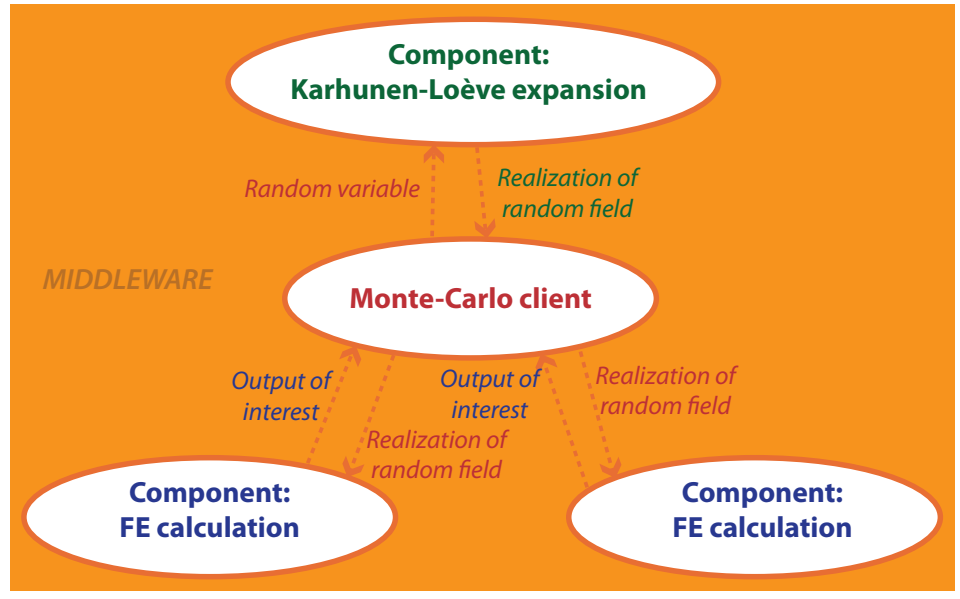

Figure 3: Schematic representation of the components architecture adapted to the MonteCarlo integration

Carlo component here acting as the "client": its main roles are to handle the random variables generation and the statistical treatment of the outputs of interest. This client is communicating with the other components, each of them being a "service". Among those services, the Karhunen-Love component synthesizes random field realizations according to a set of random numbers - provided by the client. The second main services are the FE simulators, which stand for the deterministic computations along the different random field realisations. Although only one Karhunen-Love expansion component is invoked, the FE components may be instantiated more than once, leading to a natural parallelization of the whole Monte-Carlo integration process. Such multiple instantiation can be done on a multi-processor computer or on a cluster through a network. Using the CTL, the latter may be established using any communication protocol as MPI, Pipe or TCP/IP. Finally it is worth noting that the different components shown in this ar- 
chitecture are written using different languages: $\mathrm{C}++$ for the client and the Karhunen expansion, Fortran 77 for the FE simulator (FEAP). Communications issues between those components (in particular typing issues) are all handled by the CTL middleware, leading to a very efficient and easy way to deploy architecture.

\section{Young modulus modelling - random fields}

As explained in $§ 3$, the key step to carry out the Monte-Carlo integration is to generate an important number of realizations of the Young modulus random field. Although these realizations must be independent, they are all spatially correlated and the synthesis process is not trivial. A very efficient way to achieve it is to employ the Karhunen-Loève expansion as explained by Spanos and Ghanem (2002).

\subsection{Modal decomposition}

The key idea of the Karhunen-Loève expansion is to expand any secondorder random field $f(\vec{x}, \omega)$ into a serie $(3)$. Each term of this serie is a product

of two terms, the first one depending on the spatial variable $\vec{x}$ and the second one depending on the stochastic variable $\omega$. Thus, the main feature of such a decomposition is the separation of variable.

$$
f(\vec{x}, \omega)=\bar{f}(\vec{x})+\sum_{i=0}^{+\infty} \sqrt{\lambda_{i}} \phi_{i}(\vec{x}) \xi_{i}(\omega)
$$

The first term of $(3), \bar{f}(\vec{x})$, is the expectation of the random field. The stochastic dependency appears through the random variables $\xi_{i}$, while the spatial dependency is introduced through the covariance kernel eigenmodes 
$\left(\lambda_{i}, \phi_{i}\right)$, where the $\lambda_{i}$ are the eigenvalues and the $\phi_{i}(\vec{x})$ are the corresponding eigenvectors, as it appears in (4), where $\operatorname{cov}_{f}(\vec{x}, \vec{y})$ is the covariance function for the random field $f$.

$$
\int_{\Omega} \operatorname{cov}_{f}(\vec{x}, \vec{y}) \phi_{i}(\vec{y}) d \vec{y}=\lambda_{i} \phi_{i}(\vec{x})
$$

Moreover, it is worth noting that the random variables $\xi_{i}$ form a set of orthonormal variables with respect to the probability measure $P(\omega)$ and thus form a basis of $L_{2}(\Omega)$. Finally, dealing with Gaussian random fields, the $\xi_{i}$ are Gaussian random variables as well and, being uncorrelated - or orthogonal - they are independent. Thus (3) provides a very efficient way to synthesise correlated random fields realizations, independent from one another.

\subsection{Generalised eigenvalues problem}

Considering the generalised eigenvalues problem (4), we are interested in determining the largest eigenvalues (Newman, 1996). A convenient way to achieve this is to perform a FE discretisation of (4) (see Keese (2003)), leading to the discrete generalised eigenvalues problem (5), where $\mathbb{M}$ is the mass matrix with a unitary mass density and $\mathbb{C}$ is the covariance matrix.

$$
\mathbb{M} \mathbb{C M} \vec{\phi}=\lambda \mathbb{M} \vec{\phi}
$$

This numerical problem is very alike to eigenvalues problems encountered in structural dynamics. Therefore, a Finite Element code has been used to determine the Karhunen-Loève eigenmodes by re-using the FEAP routines with the appropriate matrixes. It is worth noting that, even though $\mathbb{M}$ is a sparse matrix - due to the FE properties - the covariance matrix $\mathbb{C}$ is a full 
one. This point may lead to some difficulties linked to storage and memory requirements.

Once a set of the largest eigenvalues, as well as the corresponding eigenvectors, has been determined, the truncated Karhunen-Loève expansion enables the approximation (6) for any second-order random field. In this approximation $m$ is the number of modes which are considered and it can be shown that (6) is optimal along $m$ (Karhunen, 1947; Loève, 1978). Assuming the random field to be Gaussian, the random variables $\xi_{i}$ are normally distributed with zero expectation and variance equal to one. This series truncation introduces an error in the field approximation, which decreases when $m$ increases.

$$
\gamma_{i j}=\bar{\gamma}_{i}+\sum_{k=1}^{m} \sqrt{\lambda_{k}} \phi_{k i} \xi_{k j}
$$

In Equation (6), $\gamma_{i j}$ is the expression of the approximated random field for each node of the spatial mesh, denoted by the subscript $i$ (spatial dependency), and for each realization, denoted by the subscript $j$ (stochastic dependency). The subscript $k$ refers to the eigenmodes number: the smaller $k$ is, the bigger the corresponding eigenvalue $\lambda_{k}$. The deterministic field $\bar{\gamma}$ is the expected value of the Gaussian random field in every node of the mesh.

It is worth noting that the Karhunen-Loève expansion is carried out using the covariance function only. Among the different ways of defining a covariance model, a very simple one is chosen and described next. 


\subsection{Covariance function and correlation length}

Each term $C_{i j}$ of the covariance matrix $\mathbb{C}$ is the value of the covariance function computed between the two nodes $i$ and $j$ of the spatial mesh, whose position is given by $\vec{x}_{i}$ and $\vec{x}_{j}$. The expression of the covariance function appears in equation (7) and introduces both the variance $V$ of the Gaussian random field and the correlation length $L_{c}$. This last parameter is quite interesting to investigate: the higher the correlation length is, the stronger the statistical correlation between the value of the field in two points is as well. For a very large value of $L_{c}$, the random field tends to the deterministic case. As a matter of fact, this parameter has not been much investigated yet in the literature (Vu and Stewart, 2005), so that we propose to investigate a large range of values for this correlation length.

$$
C_{i j}=V \exp \left(-\frac{\left\|\vec{x}_{i}-\vec{x}_{j}\right\|^{2}}{L_{c}^{2}}\right)
$$

The covariance function in (7) has been chosen because it is differentiable in 0 . For the application concerned by this paper, it has been decided to consider different correlation lengths with regards to the direction of the correlation. Indeed, it may be assessed that the structure construction process has a large influence on the correlation, and especially one can assume that the correlation might be important within one batch of concrete and rather low between several batches, even with the same concrete mix design. Therefore the three spatial directions are distinguished. The vertical correlation length $L_{z}$ is related to the different steps in the building of the vessel. The radial correlation length $L_{r}$ and the orthoradial one $L_{\theta}$ are also introduced. The former is expected to be rather important with regards to the geometry 
of the considered structure. The orthoradial correlation length stands for the correlation along the cylinder perimeter. Considering these three correlation lengths leads to the definition of the covariance function given in (8).

$$
C_{i j}=V \exp \left(-\frac{\left(z_{i}-z_{j}\right)^{2}}{L_{z}^{2}}-\frac{\left(r_{i}-r_{j}\right)^{2}}{L_{r}^{2}}-\frac{R^{2}\left(\theta_{i}-\theta_{j}\right)^{2}}{L_{\theta}^{2}}\right)
$$

Equation (8) is the covariance between nodes $i$ and $j$, for which $\left(r_{i}, \theta_{i}, z_{i}\right)$ are the cylindrical coordinates of node $i$ (id. for node $j$ ). $R$ is the mean radius for the structure $(22.95 \mathrm{~m})$.

\subsection{Lognormal random field and underlying Gaussian case}

The Karhunen-Loève expansion is a very useful tool when aiming at generating Gaussian random fields. In such a case, the uncorrelated random variables $\xi_{i}$ are normally distributed and, so, are mutually independent. Nevertheless, the Gaussian scenario is not valid for a non-negative parameter like the Young modulus. Therefore we consider another very general and positive distribution which is the lognormal distribution and the Kharhunen-Loève expansion is used to generate an underlying Gaussian random field $\gamma(\vec{x}, \omega)$. The required lognormal random field $k(\vec{x}, \omega)$ can then be written as in equation (9).

$$
k(\vec{x}, \omega)=\exp (\gamma(\vec{x}, \omega))
$$

In order to compute the Karhunen-Loève expansion of $\gamma(\vec{x}, \omega)$, its covariance function is required. It can be showed that the first and second statistical moments of the Gaussian field can be derived from those of the lognormal field $k(\vec{x}, \omega)$ (see equations (10) and (11), demonstrated in Colliat 
et al. (2007)). In these equations, $\bar{k}$ is the expected value of the lognormal field, $\sigma_{k}$ and $L_{k}$ are its standard deviation and its correlation length. $\bar{\gamma}, \sigma_{\gamma}$ and $L_{\gamma}$ are the expected value, the standard deviation and the correlation length of the Gaussian field, calculated so that the lognormal field deduced from the Gaussian one has the proper characteristics. The expression of the correlation length for the gaussian random field from the one for the lognormal field is given in (12) and depends on the choice of the correlation function (see proof of the expression in de Larrard (2010)).

$$
\begin{gathered}
\bar{\gamma}=\ln \left(\frac{\bar{k}}{\sqrt{1+\sigma_{k}^{2} / \bar{k}^{2}}}\right) \\
\sigma_{\gamma}^{2}=\ln \left(1+\frac{\sigma_{k}^{2}}{\bar{k}^{2}}\right) \\
L_{\gamma}=\left(\ln \left(\frac{1}{\sigma_{\gamma}^{2}} \ln \left(1+\frac{\sigma_{k}^{2}}{\bar{k}^{2}} \exp \left(-\frac{1}{L_{k}^{2}}\right)\right)\right)\right)^{-1 / 2}
\end{gathered}
$$

The theoretical background of the Karhunen-Loève expansion can be carried out to calculate the eigenmodes of the covariance kernel. Once these modes are in hands, the expansion can be exploited as many times as necessary to compute the random fields realizations required for the Monte-Carlo Method.

\subsection{Calculation of eigenmodes}

With regards to the geometry of the structure, it can be considered that the correlation is very strong along the wall thickness. Therefore, we assume the radial correlation length to be quite large in comparison to the thickness 
of the wall $(5 \mathrm{~m})$. The lack of knowledge concerning the correlation in a concrete structure led to consider several values for the orthoradial and vertical correlation lengths, so as to investigate the influence of these parameters on the variability of the outputs of interest. Three values are considered for each correlation length: 15, 30 and $60 \mathrm{~m}$ for the orthoradial correlation length, and 10, 20 and $40 \mathrm{~m}$ for the vertical one. These values seem to be reasonable with regards to the geometry of the structure. The expected value for the Young modulus field is $26.7 \mathrm{GPa}$ and the standard deviation is $4 \mathrm{GPa}$, corresponding to a coefficient of variation of $15 \%$ for the input parameters of the problem. This order of magnitude for the coefficient of variation is the upper bound value which can be found in the literature (de Vasconcellos et al., 2003; Vu and Stewart, 2005; Berveiller et al., 2007). It also is in good accordance with the early experimental results of the APPLET project (Poyet and Torrenti, 2010; de Larrard et al., 2009).

Figure 4 shows some examples of the eigenvectors computed for a $15 \mathrm{~m}$ orthoradial correlation length and $10 \mathrm{~m}$ vertical correlation length. The modes are represented on the outer nodes of the spatial mesh for half a perimeter.

It is interesting to notice on Figure 4 that the eigenvector's frequency increases with the rank of the mode. Thus, considering a random field with small correlation lengths, the high rank modes (high frequency modes) have more importance than in the case of a random field with a high spatial correlation. This is illustrated on Figure 5 where one can observe the eigenvalues decrease as the modes rank increases. The three cases represented on Figure 5 stands for 15, 30 and $60 \mathrm{~m}$ of orthoradial correlation length and are all 


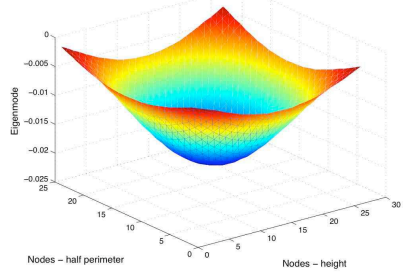

(a) Mode 1

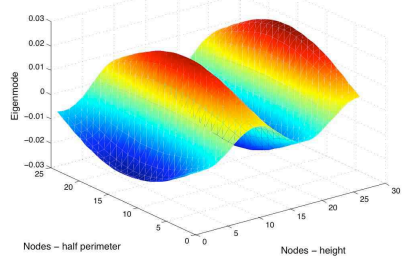

(d) Mode 9

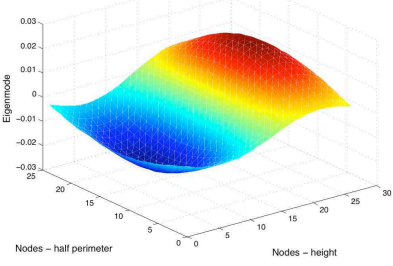

(b) Mode 2

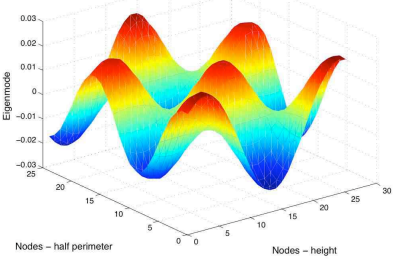

(e) Mode 16

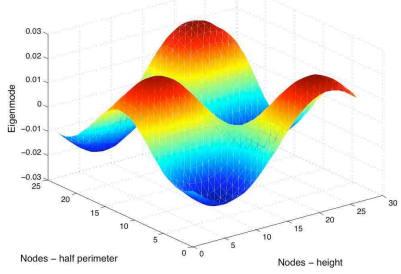

(c) Mode 8

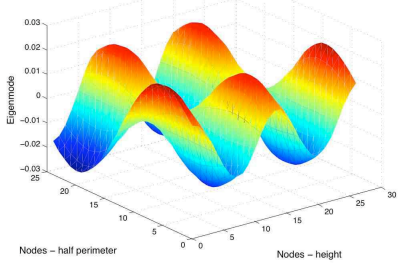

(f) Mode 17

Figure 4: Karhunen-Loève expansion eigenvectors on a half-perimeter of the structure with an orthoradial correlation length of $15 \mathrm{~m}$ 
calculated for a vertical correlation length equal to $10 \mathrm{~m}$. It appears that when the correlation length is more important, the decrease rate is more important as well, which enhances the prevalence of low frequency modes for high correlation random fields.

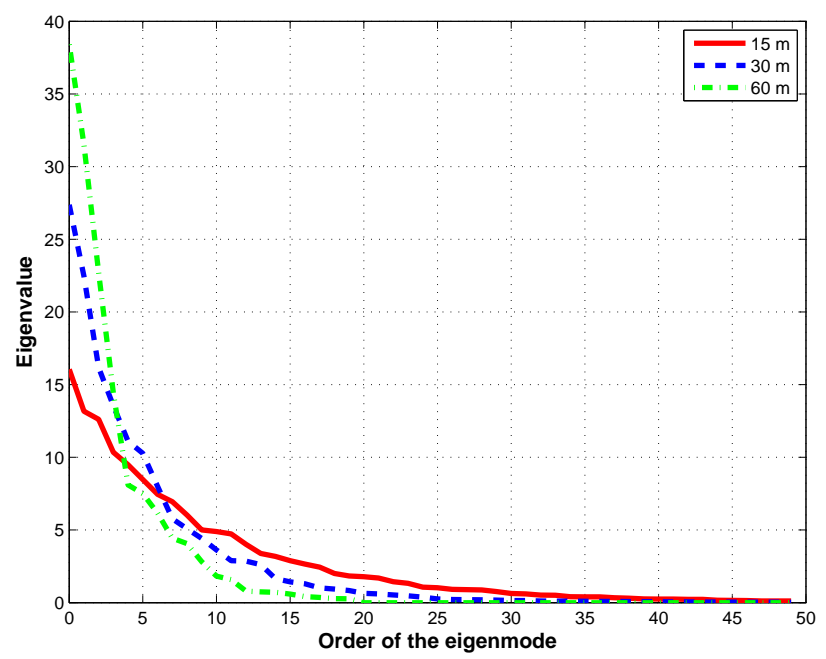

Figure 5: Eigenvalues decrease rate according to the orthoradial correlation length

As it appears in equation (6), the Karhunen-Loève series is truncated and only the $m$ first modes are retained for the random field generation. The determination of $m$ can be done thanks to the eigenvalues decrease rate. As illustrated on Figure 5, the high rank modes influence on the random field generation can be neglected when the corresponding eigenvalues are small compared to the first one. The criterion used for the random field generation has been that the modes for which the eigenvalue is less than $1 \%$ of the first mode eigenvalue can be neglected. This leads to a value of $m$ ranging from 20 to 80 according to the considered correlation length.

Once the Kharhunen-Loève expansion eigenmodes are determined, it is 
possible to generate as many realizations of the random field as requested to carry out the Monte-Carlo Method. For every Young modulus realization, a corresponding mechanical calculation is performed and the outputs of interest (maximal orthoradial stress and maximal Mazars equivalent strain) are registered for an appropriate data treatment.

\section{Results and analysis}

In order to investigate the influence of the correlation length on the variability of the outputs of interest, five probabilistic cases are considered, so that the results for three different vertical correlation lengths - with the same orthoradial correlation length - can be compared, as well as three different orthoradial correlation lengths - with the same vertical correlation length. For each case, 200 Monte-Carlo integration points are performed corresponding to 200 Young modulus random field realizations.

\subsection{Verification of the random field characteristics}

The first step in the data treatment is to verify whether the random field, generated thanks to the Karhunen-Loève expansion, has the required first and second order characteristics. This verification is satisfactory and shows that enough modes for the truncated expansion are kept. It is also necessary to check the correlation length, which is done by calculating the covariance of the field for each node of the mesh and observing the covariance decrease along different spatial directions. This is illustrated on Figure 6: from one point of the mesh, the covariance is calculated at every point with the same radial and vertical coordinates $(r, \theta)$. This covariance is normed, which means it is divided by the value of the covariance at distance of zero (which is 
verified to be equal to the required variance). Under these hypotheses, the correlation length is the distance for which the normed covariance reaches the value of $\exp (-1)=1 / e$. It appears on Figure 6 that for the three different orthoradial correlation lengths tested, the covariance reveals the expected correlation length.

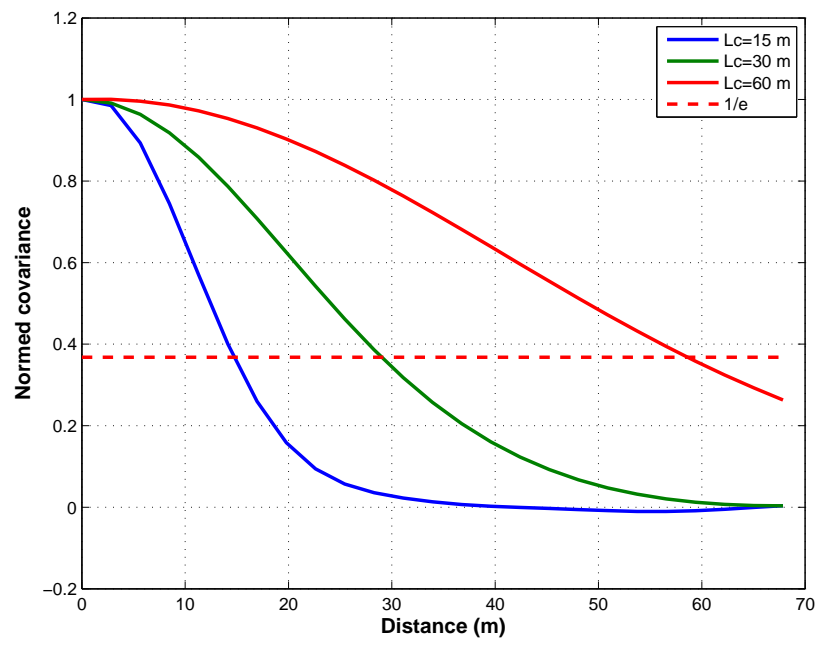

Figure 6: Normed covariance decrease according to the correlation length

After it has been verified that the input random field had the required characteristics, the variability of the outputs of interest can be observed.

\subsection{Outputs of interest confidence intervals depending on correlation lengths}

Table 1 presents the maximal orthoradial stress increase in the structure due to the Young modulus variability. Each line of the table stands for 200 realizations with the given values of orthoradial and vertical correlation lengths. For each case the radial correlation length remains equal to $5 \mathrm{~m}$. In this table one can find the mean value for the maximal orthoradial stress 
and the coefficient of variation (denoted C.V.) over the 200 realizations. The upper bound of the $95 \%$ confidence interval appears in the table (denoted Up. $95 \%)$.

Table 1: Maximal orthoradial stress (for 200 realizations)

\begin{tabular}{ccccc}
\hline \hline $\begin{array}{c}L_{\theta} \\
{[\mathrm{m}]}\end{array}$ & $\begin{array}{c}L_{z} \\
{[\mathrm{~m}]}\end{array}$ & $\begin{array}{c}\text { Mean value } \\
{[\mathrm{MPa}]}\end{array}$ & $\begin{array}{c}\text { C.V. } \\
{[\%]}\end{array}$ & $\begin{array}{c}\text { Up. 95\% } \\
{[\mathrm{MPa}]}\end{array}$ \\
\hline 15 & 10 & 11.09 & 1.87 & 11.12 \\
30 & 10 & 10.98 & 1.56 & 11.00 \\
60 & 10 & 10.86 & 1.22 & 10.87 \\
30 & 20 & 10.84 & 1.10 & 10.86 \\
30 & 40 & 10.83 & 0.94 & 10.84 \\
\hline \hline
\end{tabular}

The mean value and the upper bound of the confidence interval are to be compared with the maximum value of orthoradial stress in the deterministic case: 10.6 $\mathrm{MPa}$. The maximum mean value obtained in the probabilistic calculations is $11.1 \mathrm{MPa}$, which corresponds to a $5 \%$ increase. This increase is not very important but cannot be neglected when considering a risk of cracking under tensile stress (as the material is designed to be close to its elastic limit in case of accident). The coefficient of variation on the maximal orthoradial stress (between 1 and $2 \%$ ) should be compared with the input coefficient of variability (on the Young modulus): 15\%. It appears that the variability is absorbed by the physics of the problem. Indeed, as the loading of the structure is considered in terms of stress, the maximal stress level might not be the most accurate indicator for the influence of the Young 
modulus variability. Let us mention that the theoretical formula for the orthoradial stress in a 2-dimensional deterministic calculation does not even take the Young modulus into account. Therefore, the observation of the Mazars equivalent strain is necessary.

Table 2 presents, for the same realizations as before, the mean value, the coefficient of variation and the upper bound of the $95 \%$ confidence interval for the maximal Mazars equivalent strain over 200 realizations. This maximal mean value is equal to $782.0 \mu \mathrm{m} / \mathrm{m}$ for a deterministic calculation and can reach $929.5 \mu \mathrm{m} / \mathrm{m}$ for the probabilistic calculations, which means a $18.9 \%$ increase. The Mazars equivalent strain is an indicator correlated to the cracking risk. Indeed, the equivalent strain considers the extension strains of the material, and the mechanical damage evolution model proposed by Mazars (1986) considers that cracking occurs when the equivalent strain reaches a given threshold, itself being a random variable (which can be considered as related to the Young modulus). Therefore, such an increase seems very significant. Moreover the coefficient of variation (ranging between 10 and 12\%) is much more important than what was observed on the maximal stress and reveals that the equivalent strain is a relevant indicator for the influence of the Young modulus variability.

It appears, both in Table 1 and in Table 2, that when the correlation length increases (whatever the direction) the output of interest mean value tends toward the deterministic value, which is coherent: the higher the correlation length, the more important the decrease of the eigenvalues, so that the random field generation tends toward the expected uniform value of Young modulus. 
Table 2: Maximal equivalent Mazars strain (for 200 realizations)

\begin{tabular}{ccccc}
\hline \hline$L_{\theta}$ & $L_{z}$ & Mean value & C.V. & $\begin{array}{c}\text { Up. 95\% } \\
{[\mathrm{m}]}\end{array}$ \\
{$[\mathrm{m}]$} & {$\left[10^{-6}\right]$} & {$[\%]$} & {$\left[10^{-6}\right]$} \\
\hline 15 & 10 & 929.5 & 10.03 & 940.3 \\
30 & 10 & 895.5 & 10.21 & 906.2 \\
60 & 10 & 865.3 & 11.87 & 877.2 \\
30 & 20 & 910.5 & 11.28 & 922.5 \\
30 & 40 & 871.9 & 12.03 & 884.1 \\
\hline \hline
\end{tabular}

The next question to be answered was to determine whether there was a link between the location where the extreme values of the Young modulus (minimum and maximum) appeared in the structure, and the locations where the maximal Mazars equivalent strain was observed. Thus, 100 new calculations were run for a $15 \mathrm{~m}$ orthoradial correlation length and a $10 \mathrm{~m}$ vertical one.

\subsection{Location of Young modulus and outputs of interest extrema}

Over the 100 calculations performed for this study, Table 3 shows the mean height for the maximal and minimal Young modulus as well as for the maximal equivalent strain, and the corresponding standard deviation. It is satisfactory to verify that the Young modulus extreme values mean height is around the middle of the containment vessel, with a high standard deviation, which is in good accordance with the random field generation process. Indeed, extreme values can appear anywhere in the structure, as it is corrob- 
orated by Figure 7. This figure represents the Young modulus minimum and maximum distribution on the structure's height. On the contrary, Figure 8 shows the distribution of maximal equivalent strain vertical position. It appears that this distribution is not uniform, but the maximal values appear more often close to the top or the bottom of the structure. Indeed, the equivalent strain field for a deterministic calculation has the same shape: the values are lower in the middle of the structure and increases when getting close to the bottom or top of the containment vessel. Another calculation is performed considering only the central third of the structure, which leads to the same results. This means that the Young modulus variability introduced in the structure is sufficient to modify significantly the value of the maximal equivalent strain but not the shape of the strain field in the structure.

Table 3: Vertical position of Young modulus extreme values and maximal equivalent strain in the structure

\begin{tabular}{lcc}
\hline \hline & Mean value $[\mathbf{m}]$ & Standard deviation $[\mathbf{m}]$ \\
\hline Maximum $E$ & 23.7 & 16.3 \\
Minimum $E$ & 22.3 & 17.1 \\
\hline Maximum $\varepsilon_{e q}$ & 27.7 & 15.8 \\
\hline \hline
\end{tabular}

Table 4 indicates the distance between the point in the structure where the maximal equivalent strain is observed and the points where the Young modulus extreme values are observed. The distribution of these distances is plotted on Figure 9. It appears that no correlation exists between the position of the extreme values of the Young modulus in the structure and 


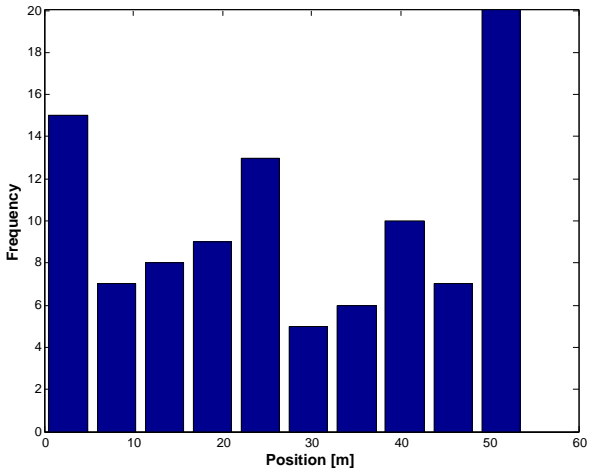

(a) Young modulus minimum

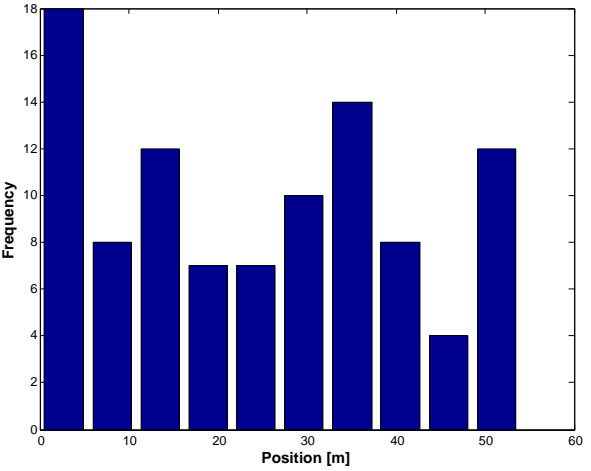

(b) Young modulus maximum

Figure 7: Distribution of vertical position of the Young modulus extreme values (100 calculations)

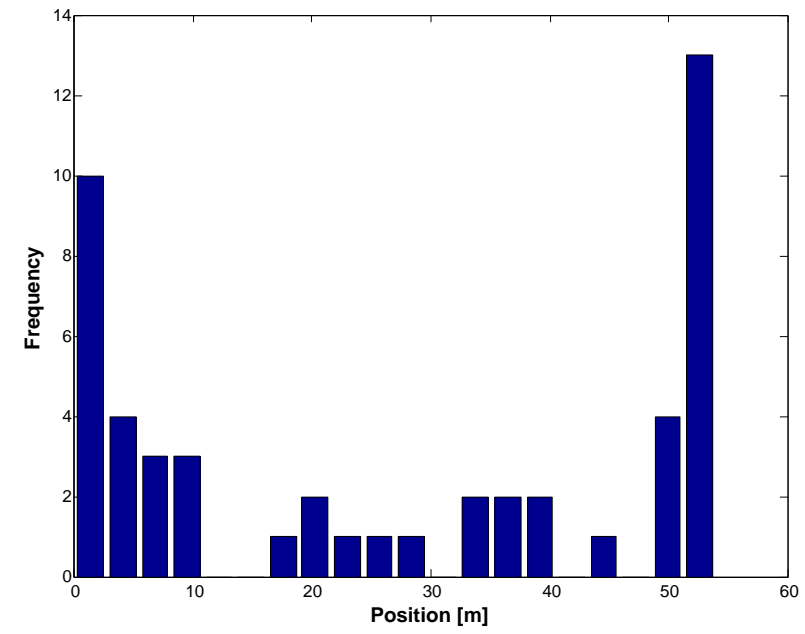

Figure 8: Distribution of vertical position of the maximal equivalent deformation (100 calculations) 
the position of the maximal equivalent strain.

Table 4: Distance between the maximal equivalent strain and the Young modulus extreme values

\begin{tabular}{lcc}
\hline \hline & Mean value $[\mathbf{m}]$ & Standard deviation $[\mathbf{m}]$ \\
\hline Maximum & 42.6 & 10.8 \\
Minimum & 29.6 & 15.1 \\
\hline \hline
\end{tabular}

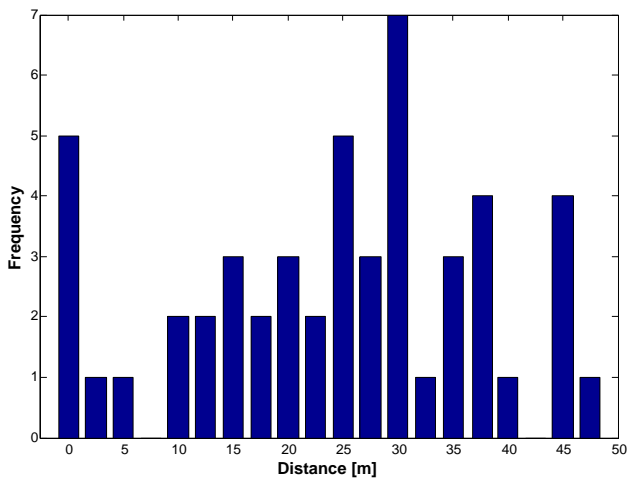

(a) Young modulus minimum

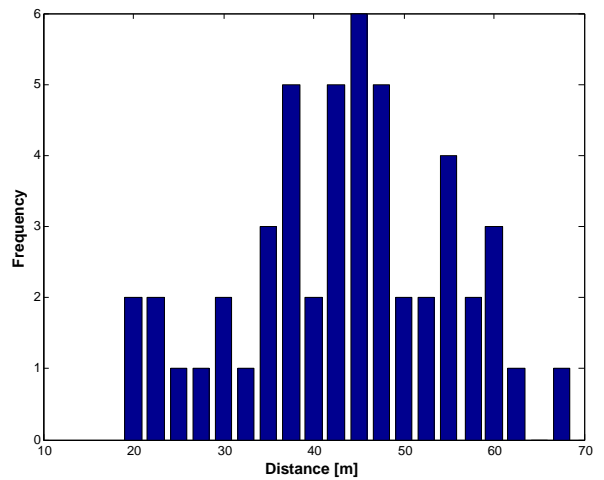

(b) Young modulus maximum

Figure 9: Distribution of distance between the maximal equivalent deformation and the Young modulus extreme values (100 calculations)

\section{Conclusion}

For this study, a simplified modelling for the mechanical behaviour of a nuclear containment vessel in case of a loss of cooling agent accident has been 
proposed, so as to carry out the Monte-Carlo Method thanks to a middleware named CTL which enables such simulations. The Young modulus random field realizations were generated with the Karhunen-Loève expansion. It has been proven that considering the Young modulus variability had a significant influence on the dimensioning strain values in comparison with deterministic calculations.

This study enhanced the influence of the correlation length on the outputs of interest variability. Therefore, the lack of information about the materials characteristics variability and the ignorance of the accurate values for the correlation length in a structure (with regards to the material formulation and the building process) turns out to be problematic to estimate the lifespan of certain structures such as nuclear containment vessels or nuclear waste storage units. This is why the APPLET project (Poyet and Torrenti, 2010) aims at quantifying the concrete variability for many mechanical characteristics (compressive and tensile strength, Young modulus, etc.) and durability

indicators (porosity, electrical resistivity, resistance to leaching attack, etc.) between several batches for a given concrete mix design as well as within one same batch. Another goal is to propose a predictive tool for the long-term behaviour of concrete structures, taking into account this variability.

\section{ACKNOWLEDGEMENT}

The investigations and results reported herein are supported by the National Research Agency (France) under the APPLET research program (grant ANR-06-RGCU-001-01). 


\section{References}

Benboudjema, F., Torrenti, J.M., 2008. Early age behaviour of concrete nuclear containments. Nuclear Engeneering and Design 238, 2495-2506.

Berveiller, M., Le Pape, Y., Sudret, B., Perrin, F., 2007. Bayesian updating of the long-term creep deformations in concrete containment vessels using a non intrusive method, in: 10th International Conference on Applications of Statistics and Probability in Civil Engineering, Tokyo, Japan.

Caflisch, R.E., 1998. Monte Carlo and Quasi-Monte Carlo Methods. Acta Numerica 7, 1-49.

Colliat, J.B., Hautefeuille, M., Ibrahimbegovic, A., Matthies, H.G., 2007. Stochastic approach to size effect in quasi-brittle materials. Comptes Rendus Mécaniques 335, 430-435.

Karhunen, K., 1947. Über lineare Methoden in des Wahrscheinlichkeitrechnung. ANN. Acad. Sci. Fennicae, Ser. Al. Math.-Phys. (in German) 37, $1-79$.

Keese, A., 2003. Numerical solution of systems with uncertainties - a general purpose framework for stochastic finite elements. Phd thesis. Technischen Universitat Braunschweig. Germany.

de Larrard, T., 2010. Etude probabiliste des propriétés du béton. Applications aux enceintes de confinement et au stockage des déchets radioactifs. (in french). Ph.D. thesis. ENS Cachan. 
de Larrard, T., Benboudjema, F., Colliat, J.B., Torrenti, J.M., Deleruyelle, F., 2009. Correlations between measures and development of a predictive model for the long-term behavior of concrete structures subjected to leaching, in: NUCPERF 2009 EFC Event 317, RILEM, Cadarache, France.

Loève, M., 1978. Probability theory. volume II of Graduate Texts in Mathematics. Springer-Verlag. 4th edition.

Matthies, H.G., Niekamp, R., Steinford, J., 2006. Algorithms for strong coupling procedures. Computer Methods in Applied Mechanics and Engineering 195, 2028-2049.

Mazars, J., 1986. A description of micro and macroscale damage of concrete. Engineering Fracture Mechanics 25, 729-737.

Metropolis, N., Ulam, S., 1949. The Monte Carlo Method. Journal of American Statistical Association 44, 335-341.

Newman, A.J., 1996. Model reduction via the Karhunen-Loeve expansion part I : an exposition. Technical report. University of Maryland, USA.

Poyet, S., Torrenti, J.M., 2010. Caractérisation de la variabilité des performances des bétons. application à la durabilité des structures (groupe de travail APPLET), in: Annales du BTP (in press), (in french).

Spanos, P.D., Ghanem, R.G., 2002. Stochastic finite element, a spectral approach. Dover Publication. revised edition.

Szyperski, C., 1998. Component Software - Beyond Object-Oriented Programming. Addison-Wesley and ACM Press. 
Taylor, R.L., Zienkiewicz, O.C., 2005. The finite element method. Elsevier, Oxford. 6th edition.

de Vasconcellos, M., Campos Filho, A., Roberto Maestrini, S., 2003. Response variability in reinforced concrete structures with uncertain geometrical and material properties. Nuclear Engeneering and Design 226, $205-220$.

Vu, K.A.T., Stewart, M.G., 2005. Predicting the likehood and extent of reinforced concrete corrosion-induced cracking. Journal of Structural Engineering 131, 1681-1689. 